https://helda.helsinki.fi

\title{
Adaptation of neuromagnetic N1 without shifts in dipolar orientation
}

Campbell, Tom

2007

Campbell , T \& Neuvonen , T 2007 , ' Adaptation of neuromagnetic N1 without shifts in dipolar orientation ' , NeuroReport, vol. 18 , no. 4 , pp. 377-380 .

http://hdl.handle.net/10138/136178

submittedVersion

Downloaded from Helda, University of Helsinki institutional repository.

This is an electronic reprint of the original article.

This reprint may differ from the original in pagination and typographic detail.

Please cite the original version. 


\title{
Adaptation of neuromagnetic NI without shifts in dipolar orientation
}

\author{
Tom Campbell ${ }^{\mathrm{a}, \mathrm{b}}$ and Tuomas Neuvonen ${ }^{\mathrm{c}}$
}

\author{
${ }^{a}$ Helsinki Collegium for Advanced Studies, University of Helsinki, Finland, 'Biomag Laboratory, Engineering Centre, Helsinki University Central Hospital, \\ Helsinki, Finland and ${ }^{\mathrm{C} D e p a r t m e n t ~ o f ~ P h y s i o l o g y, ~ N e u r o s c i e n c e ~ U n i t, ~ I n s t i t u t e ~ o f ~ B i o m e d i c i n e, ~ U n i v e r s i t y ~ o f ~ H e l s i n k i, ~ F i n l a n d ~}$ \\ Correspondence to DrTom Campbell, PhD, Helsinki Collegium for Advanced Studies PO Box 4, University of Helsinki, FIN-00014, Finland \\ Tel: + 358415409764; fax: + 358919124509; e-mail: tom.campbell@helsinki.fi
}

Received 20 November 2006; accepted 29 November 2006

\begin{abstract}
306-channel magnetoencephalography, coregistered with highresolution volumetric magnetic resonance imaging, was used with 10 healthy participants to test if repetition adapts subsequent processing of sounds in a sequence and whether this adaptation influenced the orientation of the dipolar sources in the auditory cortex. Auditory $\mathrm{NIm}$ responses to I $\mathrm{kHz}$ pure tones were indexed by clusters of sensors situated bilaterally over the temporal lobes.
\end{abstract}

$\mathrm{NIm}$ was augmented in amplitude at an interstimulus interval of $16 \mathrm{~s}$ relative to $\mathrm{Is}$. This neuromagnetic amplitude augment occurred in dipoles in the vicinity of the auditory cortex, without significant shifts in the dipolar orientation. Recent repetition thus adapts auditory cortical neurons, in a manner subject to recovery after a period of silence. NeuroReport 18:377-380 (C) 2007 Lippincott Williams \& Wilkins.

Keywords: cortical adaptation, human auditory cortex, magnetoencephalography, NIm response

\section{Introduction}

Responsivity of the brain to auditory onsets could be critical to survival. The brain processes elicited by a sound are subject to adaptation [1,2]. That is, repetition causes a reduction in the generation of those brain processes, a reduction which is termed adaptation. This adaptation manifests as a decline in responsivity of neuronal elements to stimulation over time, in a manner that is subject to recovery after a period of silence during which time those neuronal elements remain unstimulated.

Accordingly, shortening the intervening silence between tones, the interstimulus interval (ISI), could cause a neuronal adaptation that reduces the amplitude of the N1 response of the auditory event-related potential (ERP), as is derived from conventional electroencephalograms recorded from the scalp. This N1 response, the first long-latency vertex negativity of the auditory ERP, peaks ca. $100 \mathrm{~ms}$ after onset and is seen to attenuate in amplitude with shorter ISIs [3]. This attenuation of N1 tenably could be mediated via an adaptation of neuronal elements that become progressively less responsive upon repeated recent stimulation. This attenuation of $\mathrm{N} 1$ could be related to reductions in the distracting effects of ignored sound and could also have an influence upon the audio-visual processing of phonetic material [4-10].

Although there is evidence of adaptation of neuronal populations that could generate supratemporal aspects of the auditory N1 response within the auditory cortex [1-3], at ISIs as short as $1 \mathrm{~s}$, a distinct aspect of the N1 response to sounds could also be generated within another superficial region of cortex [11-13]. Scalp current density analyses indicate that this region is focal to anterior-frontal rather than temporal regions of the brain.
The possibility, however, remains that this seemingly anterior frontally generated N1 component could rather have reflected a dipolar supratemporal source, which shifted in orientation [14] with reductions in ISI. Accordingly, supratemporal dipolar sources of auditory N1 would exhibit a shift in orientation in the sagittal plane as a function of ISIs within the range 1-16 s. Such dipole models may be appropriately derived with the magnetically measured counterpart of the supratemporal N1, the N1m, as derived from magnetoencephalogram (MEG) [14]. Increasing the ISI from 1 to $16 \mathrm{~s}$ would thus influence the orientation of the $\mathrm{N} 1 \mathrm{~m}$ dipole. We term this the dipole orientation hypothesis.

Neuronal elements in the vicinity of the auditory cortex could be adapted by recent activation in response to a repeated sound. Those neuronal elements could thus become less responsive upon subsequent repetitions, in a manner subject to recovery when those elements are not stimulated, as occurs after a period of silence. Increasing the ISI from 1 to $16 \mathrm{~s}$ would thus enhance the amplitude of the N1m dipole. We term this the adaptation hypothesis.

\section{Materials and methods}

Ten healthy participants (five women, aged 24-31 years; mean 28 years, 10 months) volunteered with informed written consent in accordance with the Declaration of Helsinki with hospital ethical committee approval. All were right-handed and reported intact hearing and corrected-tonormal or normal vision. Volunteers were comfortably seated in an acoustically shielded and magnetically shielded room (ETS-Lindgren Euroshield Oy, Eura, Finland) where they watched a self-chosen subtitled film presented without the soundtrack. 
Participants were instructed to ignore the auditory stimuli [1-kHz tone bursts, $85-\mathrm{dB}(\mathrm{A}), 50 \mathrm{~ms}$ duration, $10-\mathrm{ms}$ rise and fall time] that were delivered by Presentation software (Neurobehavioral Systems, Albany, California, USA) binaurally via plastic tubes and small soft ear pieces. The independent variable of interest was the ISI from the beginning of the stimulus to the beginning of the next stimulus and had two levels (1 s, 16s). Each of the 10 stimulus runs was composed of 250 or 60 stimulations delivered at constant ISI of 1 or $16 \mathrm{~s}$, respectively. Runs were separated by $120 \mathrm{~s}$. ISI was alternated on successive runs, and the choice of the initial ISI was counterbalanced across participants.

MEG (VectorView 306-channel system, Elektra Neuromag Oy, Finland) was acquired in the band $0.10-172.2 \mathrm{~Hz}$ and digitized at a rate of $600.6 \mathrm{~Hz}$. Following artefact rejection (excluding responses with EOG $\pm 50 \mu \mathrm{V}$ ), MEG responses for each ISI were averaged (disregarding the first sound from each block). Averaged responses were digitally filtered offline with a passband of $1-30 \mathrm{~Hz}$ and baseline-corrected in relation to the mean amplitude at a $100-\mathrm{ms}$ prestimulus interval. In addition to temporal filtering, signal space projection was applied to suppress external magnetic noise $[15,16]$.

Response amplitudes were then quantified from the averaged response to the stimuli using equivalent current dipoles (ECD) fitted in a least-squares sense at the individually determined peak latency of the $\mathrm{N} 1 \mathrm{~m}$ response using a subset of sensors over the temporal area of each hemisphere, each subset of which consisted of 21 sensor triplets. Each triplet was constituted by a magnetometer alongside a pair of planar gradiometers. Triplets were identical to those used in a previous N1m investigation [17].

A three-dimensional reconstruction of each brain's surface - as derived from high-resolution T1-weighted volumetric magnetic resonance images that were acquired with a 1.5-T Siemens Magnetom (Erlangen, Germany) $(n=8)$ or 3-T Philips Achieva (Best, The Netherlands) $(n=2)$ - was used to individually determine the optimal location of the centre of the single sphere used during this ECD fitting for each individual.

This sphere was coregistered with nasion, left-periauricular and right-periauricular points as was digitized and subsequently derived from the digitized position of head position indicator coils used to determine the relative position of the head to the sensors during the MEG measurement. This sphere was that, in sagittal, axial and coronal planes, which best-fitted, in the least-squares sense, points that traced a smooth curve parallel to the skull upon the brain's surface, when there were roughly 20 points plotted upon one single slice oriented in each of these three planes. The single sphere for each individual, used for ECD fitting for that individual, included both hemispheres and was close to symmetrically positioned upon all slices in each plane.

Differences in the dipolar amplitudes, latencies, moments, orientations and locations were then tested with ISI $(1 \mathrm{~s}, 16 \mathrm{~s}) \times$ hemisphere (left, right) repeated-measures analysis of variance.

\section{Results}

As illustrated for the right hemisphere of an individual participant in Fig. 1, dipoles were localized within the vicinity of the auditory cortex. As depicted in Fig. 2, in all volunteers, the amplitude of $\mathrm{N} 1 \mathrm{~m}$ responses to the tones was significantly suppressed bilaterally at the $1 \mathrm{~s}$ relative to the 16 s ISI, $P<0.01$. This suppression of ECD amplitude was accompanied by a reduction in ECD latency at

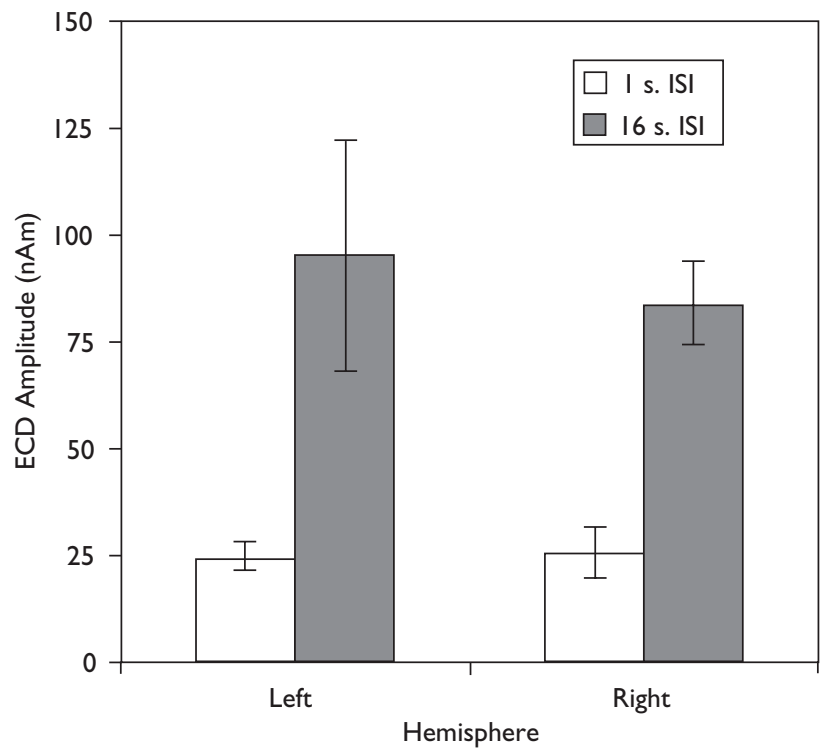

Fig. 2 Mean $( \pm S E M)$ amplitude of $\mathrm{NIm}$ as a function of interstimulus interval and hemisphere.

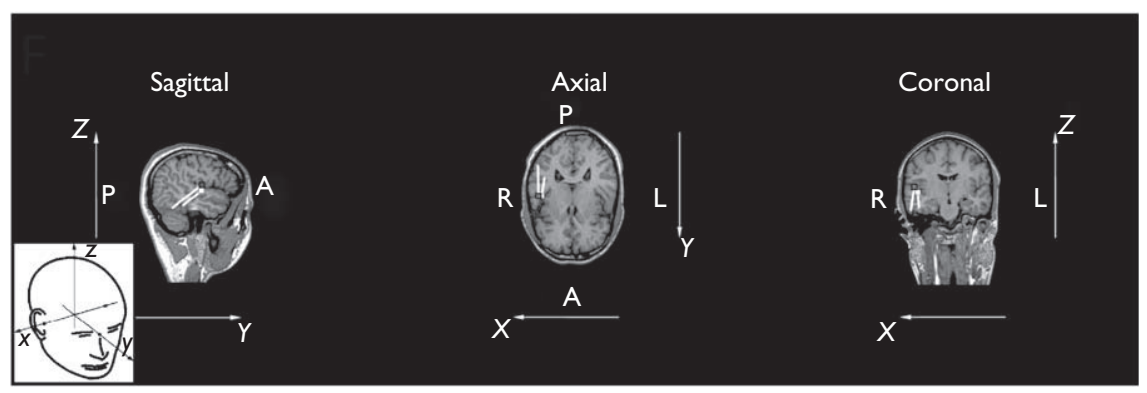

Fig. I Dipole locations in the right hemisphere of an individual participant as a function of interstimulus interval (white I s; grey 16 s) superimposed onto the participant's magnetic resonance images. Lines denote dipolar amplitudes and orientation. L, left hemisphere; R, right hemisphere; A, anterior; $\mathrm{P}$, posterior. The coordinate system is depicted within the axes of each plane and the inset (inset adapted from Figure 5.2 within and reprinted with permission from [18]). 
Table I Mean ( \pm SEM) location of NIm dipoles as a function of hemisphere and ISI

\begin{tabular}{lcccc}
\hline ISI & Hemisphere & $|x|^{\mathrm{a}}$ & \multicolumn{1}{c}{$y$} & $\mathrm{z}$ \\
\hline Is & Left & $41.6 \pm 3.1$ & $6.0 \pm 3.9$ & $53.4 \pm 4.8$ \\
Is & Right & $49.4 \pm 1.8$ & $10.5 \pm 3.7$ & $55.5 \pm 4.5$ \\
Is & Mean & $45.7 \pm 1.9$ & $8.4 \pm 2.7$ & $54.5 \pm 3.2$ \\
I6s & Left & $40.6 \pm 3.6$ & $9.3 \pm 5.0$ & $52.3 \pm 3.7$ \\
I6s & Right & $41.7 \pm 2.2$ & $9.0 \pm 3.3$ & $52.9 \pm 6.0$ \\
I6s & Mean & $41.2 \pm 2.0$ & $9.1 \pm 2.8$ & $52.6 \pm 3.6$ \\
\hline
\end{tabular}

A right-handed Cartesian coordinate system is used relative to the centre of the sphere in which the direction of $x$ is from left to right periauricular point, the $y$-axis points upward through the vertex and the $z$-axis points forward through nasion. The modulus of $x$-location was used.

ISI, interstimulus interval.

${ }^{\mathrm{a} A} \mathrm{~A} x e s$ with significant effects of ISI.

Table 2 Mean $( \pm$ SEM) clockwise positive angle of NIm dipoles from a reference moment pointing in the positive direction of the vertical axis of Fig. I upon the different planes as a function of hemisphere and ISI

\begin{tabular}{lcccl}
\hline ISI & Hemisphere & Sagittal & Axial & Coronal \\
\hline Is & Left & $223.4 \pm 13.3$ & $202.1 \pm 8.8$ & $212.3 \pm 18.3$ \\
Is & Right & $216.7 \pm 7.6$ & $181.6 \pm 26.4$ & $162.6 \pm 7.2$ \\
Is & Mean & $219.9 \pm 7.3$ & $191.3 \pm 14.3$ & $186.1 \pm 10.9$ \\
I6s & Left & $210.4 \pm 6.2$ & $170.7 \pm 16.1$ & $166.7 \pm 9.6$ \\
I6s & Right & $223.5 \pm 7.1$ & $190.5 \pm 11.5$ & $186.9 \pm 10.8$ \\
I6s & Mean & $217.7 \pm 4.9$ & $181.7 \pm 9.6$ & $177.9 \pm 7.5$ \\
\hline
\end{tabular}

This reference moment pointed upwards $\left(Q_{y} Q_{z}\right)$ in the sagittal $(y, z)$, forwards $\left(Q_{z}, Q_{y}\right)$ in the axial $(x, z)$ and upwards $\left(Q_{x}, Q_{z}\right)$ in the coronal $(x, z)$ plane. ISI, interstimulus interval.

Table 3 Mean $( \pm S E M)$ NIm dipolar moments as a function of hemisphere and ISI

\begin{tabular}{lcccc}
\hline ISI & Hemisphere & $Q_{x}$ & $Q_{y}{ }^{a}$ & $Q_{z}{ }^{a}$ \\
\hline Is & Left & $-5.5 \pm 2.8$ & $-I I .2 \pm 1.7$ & $-15.6 \pm 4.1$ \\
Is & Right & $8.5 \pm 4.1$ & $-15 . I \pm 4.7$ & $-15.7 \pm 2.8$ \\
Is & Mean & $1.9 \pm 3.0$ & $-13.2 \pm 2.6$ & $-15.6 \pm 2.4$ \\
I6s & Left & $25.8 \pm 26.7$ & $-41.4 \pm 16.6$ & $-64.2 \pm I I . I$ \\
I6s & Right & $-4.7 \pm I I . I$ & $-52 \pm 10 . I$ & $-49.8 \pm 7.9$ \\
$\mathrm{I6s}$ & Mean & $8.9 \pm 13.4$ & $-47.3 \pm 9.0$ & $-56.2 \pm 6.6$ \\
\hline
\end{tabular}

ISI, interstimulus interval.

${ }^{a}$ Moments with significant effects of ISI.

$1 \mathrm{~s}$ relative to the $16 \mathrm{~s}$ (ISI $1 \mathrm{~s}: 85.9 \mathrm{~ms} \pm 1.6<$ ISI $16 \mathrm{~s}$ : $93.3 \pm 2.7 \mathrm{~ms}), P<0.05$, coupled with a modest shift along the $x$-axis (Fig. 1, inset [18]) from a medial to lateral location, $P<0.05$ (Table 1 ). The $y$ and $z$ locations, however, did not differ significantly with ISI, F $<1.1$, nor did the angles denoting the orientation of dipole differ significantly with ISI, neither in the sagittal, axial nor coronal planes (Table 2). Significant influences of ISI on dipole moments, $Q_{y}, P<0.05$, and $Q_{z}$ (Table 3), $P<0.0001$, were accordingly not because of shifts in dipolar orientation, but rather a bilateral increase in the amplitude of dipoles that were oriented primarily along the sagittal plane. All other main effects and interactions were nonsignificant.

\section{Discussion}

The results showed that recent repetition of a sound adapted the N1m response, which can be explained by dipolar sources within the vicinity of the auditory cortex. That is, a reduction in ISI from 16 to $1 \mathrm{~s}$ attenuated the amplitude of the $\mathrm{N} 1 \mathrm{~m}$ response, as can be explained by a dipole that orients primarily sagittally in the vicinity of the auditory cortex (Fig. 1). This finding corroborates the adaptation hypothesis that, within the auditory cortices bilaterally, neuronal elements, which are responsive to sound, are adapted following recent activation such that those elements become less responsive upon subsequent repetitions, in a manner which is subject to recovery after a period of silence.

These temporal lobe dipoles did not shift in orientation with ISI, despite a significant increase in amplitude. The adaptation hypothesis thus was supported, although the dipole orientation hypothesis thus went unsupported. In turn, it remains tenable that the anterior frontal N1 [11-13] which could be obligatorily responsive to stimuli presented at ISIs less than $8 \mathrm{~s}-$ is generated within the frontal lobes.

What nobody understands is why a decrease in ISI caused the temporal dipoles to shift from more medial to lateral locations. Such a finding warrants independent replication. Nor is it fully understood why the latency of N1m generation was prolonged at longer ISIs. This prolongation, however, replicated previous auditory N1 results [11].

At long ISIs, a tonal sound led to the dipolar generation of a prolonged and augmented N1m that could implicate a stronger relative contribution from the primary auditory cortex. At shorter ISIs, conversely, nonprimary auditory areas lateral to the primary auditory cortices $[19,20]$ could exert a stronger relative contribution. Accordingly, the location of the dipole shifted laterally with a reduction in ISI.

Clues to how this shift and its consequences relate to brain function could reside within the effects of auditory distraction upon cognitive performance and the effects of the to-be-ignored background sounds upon the generation of evoked responses. A fleeting disruption of the categorization of visually presented digits (odd-versus-even) that occurs when large populations of nonadapted afferent auditory elements are activated would seem to be confined to the detection of rare auditory onsets; for example, when the ISI between auditory stimuli is long [1]. This distraction away from the primary task towards such a discontinuity in the auditory environment could have evolved to promote survival within hostile settings $[1,2]$.

By contrast, a subtler and distinct form of auditory distraction exists that disrupts performance upon cognitive tasks with a requirement for the temporary retention of information in memory exists [4-6]. Even when the initial onset of the auditory sequence has already been detected, such cognitive performance is still disrupted by background sound. This distraction effect may be related to the preattentive perceptual processing of the sequence of tobe-ignored sounds. This effect still occurs when N1 has undergone considerable adaptation [4-5], which, in this investigation, is associated with medial-to-lateral dipole location shifts. This distraction effect increases alongside the amplitude of an anterior frontal N1 component [4-5], a component which could be generated focally within the frontal lobes [11-13].

\section{Conclusion}

Recent repetition of sound adapts auditory cortical neurons, in a manner subject to recovery after a period of silence. This 
adaptation reduces the N1m amplitude without influencing the orientation of the dipolar source situated within each temporal lobe in the vicinity of the auditory cortices of the human brain.

\section{Acknowledgements}

This study was financially supported by a Marie Curie Fellowship of the European Community programme, 'Improving the Human Research Potential and the SocioEconomic Knowledge Base' (HPMF-CT-2000-00902) and by the University of Helsinki.

\section{References}

1. Jääskeläinen IP, Ahveninen J, Bonmassar G, Dale AM, Ilmoniemi RJ, Levänen $S$, et al. Human posterior auditory cortex gates novel sounds to consciousness. Proc Natl Acad Sci U S A 2004; 101:6809-6814.

2. Ulanovsky N, Las L, Farkas D, Nelken I. Multiple time scales of adaptation in auditory cortex neurons. J Neurosci 2004; 24:10440-10453.

3. Hari R, Kaila K, Katila T, Tuomisto T, Varpula T. Interstimulus-interval dependence of the auditory vertex response and its magnetic counterpart: implications for their neural generation. Electroencephalogr Clin Neurophysiol 1982; 54:561-569.

4. Campbell TA, Winkler I, Kujala T, Näätänen R. The N1 hypothesis and irrelevant sound: evidence from token set effects. Cogn Brain Res 2003, 18:39-47.

5. Campbell TA, Winkler I, Kujala T. Disruption of immediate memory and brain processes: an auditory ERP protocol. Brain Res Protoc 2005; 14:77-86.

6. Campbell TA, Beaman CP, Berry DC. Auditory memory and the irrelevant sound effect: further evidence for changing-state disruption. Memory 2002; 10:199-214.

7. Jacobsen T, Schröger E. Is there pre-attentive memory-based comparison of pitch? Psychophysiology 2001; 38:723-727.

8. Jacobsen T, Schröger E, Horenkamp T, Winkler I. Mismatch negativity to pitch change: varied stimulus proportions in controlling effects of neural refractoriness on human auditory event related brain potentials. Neurosci Lett 2003; 344:79-82.

9. Campbell TA, Beaman CP, Berry DC. Changing-state disruption of lip-reading by irrelevant sound in perceptual and memory tasks. Eur J Cogn Psychol 2002; 14:461-474.

10. Jääskelainen IP, Ojanen V, Ahveninen J, Auranen T, Levanen S, Möttönen R, et al. Adaptation of neuromagnetic N1 responses to phonetic stimuli by visual speech in humans. Neuroreport 2004; 15:2741-2744.

11. Alcaini M, Giard MH, Thévenet M, Pernier J. Two separate frontal components in the N1 wave of the human auditory-evoked response. Psychophysiology 1994; 31:611-615.

12. Giard MH, Perrin F, Echallier JF, Thevenet M, Froment JC, Pernier J. Dissociation of temporal and frontal components in the human auditory N1 wave: a scalp current density and dipole model analysis. Electroencephalogr Clin Neurophysiol 1994; 92:238-252.

13. Alcaini M, Giard MH, Echallier JF, Pernier J. Selective auditory attention effects in tonotopically-organized cortical areas; a topographic ERP study. Hum Brain Mapp 1995; 2:159-169.

14. Cansino S, Ducorps A, Ragot R. Tonotopic cortical representation of periodic complex sounds. Hum Brain Mapp 2003; 20:71-81.

15. Tesche CD, Uusitalo MA, Ilmoniemi RJ, Huotilainen M, Kajola M, Salonen O. Signal-space projections of MEG data characterize both distributed and well-localized neuronal sources. Electroencephalogr Clin Neurophysiol 1995; 95:189-200.

16. Uusitalo MA, Ilmoniemi RJ. Signal-space projection method for separating MEG or EEG into components. Med Biol Eng Comput 1997; 35:135-140.

17. Shestakova A, Brattico E, Soloviev A, Klucharev V, Huotilainen M Orderly cortical representation of vowel categories presented by multiple exemplars. Cogn Brain Res 2004; 21:342-350.

18. Neuromag Oy. Source modelling software user's guide software version 5.2.4 2001

19. Hall DA, Hart HC, Johnsrude IS. Relationships between human auditory cortical structure and function. Audiol Neuro-Otol 2003; 8:1-18.

20. Viceic D, Fornari E, Thiran J-P, Maeder PP, Meuli R, Adriani M, Clarke S. Human auditory belt areas specialized in sound recognition: a functional magnetic resonance imaging study. Neuroreport 2006; 17:1659-1662. 